\title{
Utilization of Epigastric Vessels in Combined Penile Straightening and Revascularization Surgery
}

\author{
Özer Ural Çakıcı ${ }^{1 *}$, Emrah Okulu² and Önder Kayıgil ${ }^{3}$ \\ ${ }^{1}$ Attending Urologist, Yildirim Beyazit University Yenimahalle Training and Research Hospital, Turkey \\ ${ }^{2}$ Associate Professor in Urology, Yildirim Beyazit University Ataturk Training and Research Hospital, Turkey \\ ${ }^{3}$ Professor in Urology, Yildirim Beyazit University Ataturk Training and Research Hospital, Turkey
}

*Corresponding author: Özer Ural Çakıcı, Attending Urologist, Yildirim Beyazit University Yenimahalle Training and Research Hospital, FEBUBerdan Str. 2/3 Kurtulus - Ankara. 06600, Turkey

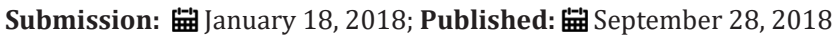

\section{Introduction}

\section{General points on the peyronie's disease}

Peyronie's disease (PD) is characterized by fibrosis of tunica albuginea. Buckling trauma to the penis is implicated as the main etiological factor. The usual clinical presentation of the disease is penile curvature, and the two phases of the disease is well known. Even though most of the patients can be managed by the medical treatments, a group of patients with severe deformity will require surgery. Surgery is only to be made in stable phase of the disease. Surgical approach is usually made by a circumcision incision and total degloving of the penis. Careful elevation of neurovascular bundle, plication of the contralateral side with or without excision, and patch repair or corporoplasty of the curvature side are the main surgical rules that had been thoroughly described in the classical texts. Several different grafts had been used such as dermis, vein, tunica vaginalis, intestine, pericardium and acellular xenografts for patch repair procedures. Due to surgical experience and relatively appropriate graft length, saphenous vein harvesting is usually performed in procedures necessitates grafting.

\section{Concurrent erectile dysfunction in peyronie's disease patients}

Erectile dysfunction concurrent with PD is posing a challenge for physicians. Before planning the treatment modality, erectile dysfunction should be carefully investigated, and it should be differentiated from inability to intercourse due to the penile deformity. Doppler ultrasound of the penis, cavernosometry and corpus cavernosum electromyography are mainstay tests of preoperative evaluation and we obtain both in every patient with erectile dysfunction in our clinical practice $[1,2]$.

\section{Surgical treatment of concurrent erectile dysfunction and peyronie's disease}

Patients with severe penile deformity with concurrent erectile dysfunction are candidates for the penile prosthesis implantation. However, a non-negligible part of patients has less severe deformity or mild vasculogenic erectile dysfunction, yet a group of patients simply questioning the physician for another option. We have already reported both the preliminary results and the longterm results of our penile revascularization case series combined with complex penile correction surgery $[1,3]$. Herein, we describe a novel surgical technique during the combination of Peyronie's surgery and penile revascularization that could be defined as harvesting epigastric vein for cavernous patch; instead of using saphenous vein. Thus, the surgical morbidity of incision and dissection in another region, while using the same paramedian incision for both artery supply and vein harvesting.

\section{Surgical technique and utilization of epigastric vessels}

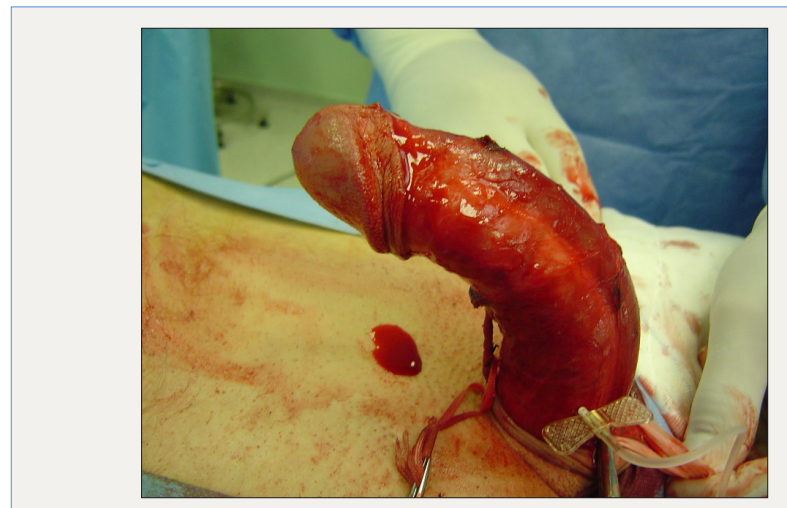

Figure 1:

The initial penile incision is usually made on the previous circumcision line. Penis is totally degloved to the base ensuring adequate hemostasis on subcutaneous tissue. An artificial erection is created with intracavernosal infusion of sterile isotonic solution (Figure 1). At this point we make a manual examination to assess curvature degree, maximum point of the curvature, and tension strength which are the determining factors for us to decide on which surgical technique we will use. We prefer using bilateral parauretral incisions to elevate neurovascular bundle, and just be- 
fore elevating the neurovascular bundles, we dissect deep dorsal vein with a dorsal midline incision to the level of the pubic bone. Having finished the neurovascular bundle dissection, the cavernous bodies are totally exposed. Then we place the plication sutures under artificial erection. Tension strength is reassessed manually while the sutures are held in their positions. Depending on the high tension on the plaque side we prefer to make a modified "Egydio" incision on the plaque (Figure 2) [4,5]. This step needs a significant care for making an incision as small as can be made, yet enough to relax the tension strength. If the cavernous defect is adequate to epigastric vein grafting, we no further need to harvest saphenous vein.

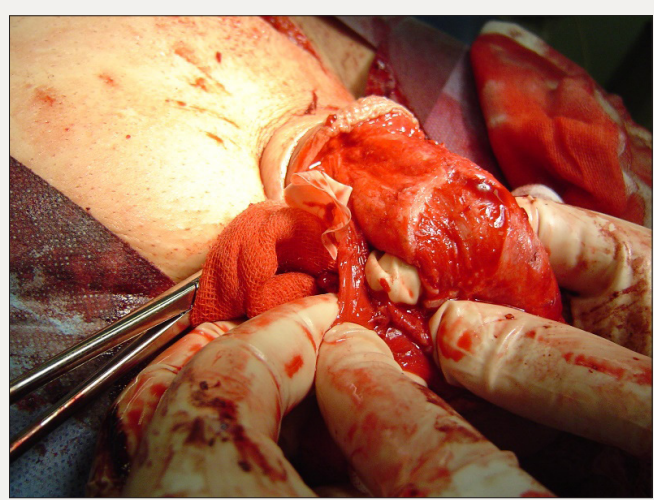

Figure 2:

A left paramedian incision reaching from inguinal area to the level of the umbilicus is made. The fascia is opened, and the epigastric vessels are found between the rectus muscle fibers. Side branches of the artery and the vein are ligated, and the main vessels are ligated at the point they are getting into to the muscle. Proximal dissection of the epigastric vessels continues with the area under the spermatic cord through the vascular sheath of the femoral vessels. Adequate vascular dissection aims freeing the epigastric artery to its originating point from femoral artery (Figure 3). The epigastric vein can be harvested at this point to be used as a vein graft (Figure 4).

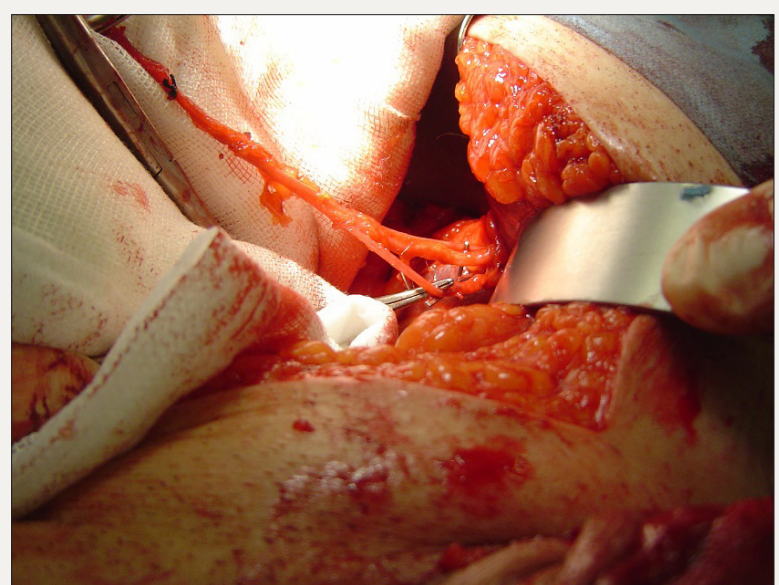

Figure 3:

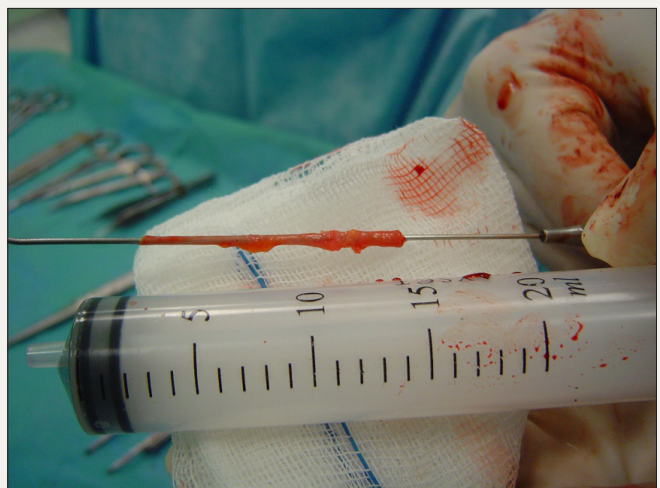

Figure 4:

The vein graft is prepared and sutured to the cavernous defect using 6/0 Vicryl sutures (Figure 5). Penile straightening is reassessed with artificial erection (Figure 6). The epigastric artery is taken to the dorsal penile area through a subcutaneous prepubic tunnel. The deep dorsal vein of the penis is ligated with a silk suture just under the pubic bone. An end-to-side anastomosis is carried out under optical enhancement between the deep dorsal vein and the epigastric artery with the modified Furlow-Fisher technique. Patency of the anastomosis is evaluated under direct vision. The Buck's fascia is reapproximated using absorbable fine sutures. The penile skin is closed with fast absorbable sutures. Penis is dressed using mild occlusive material. The paramedian incision is closed in anatomical fashion and dressed.

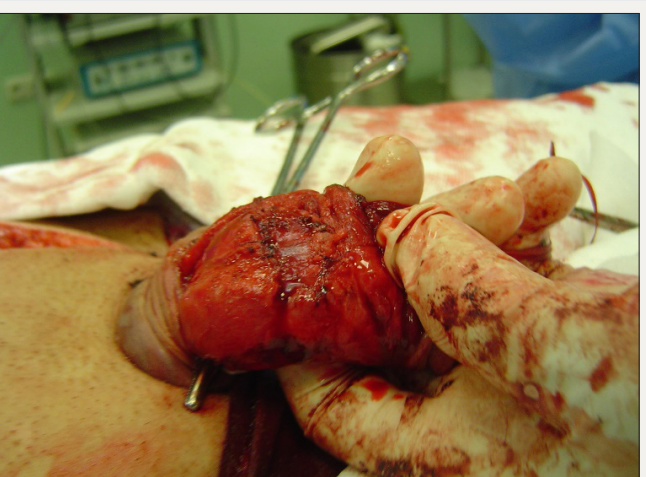

Figure 5:

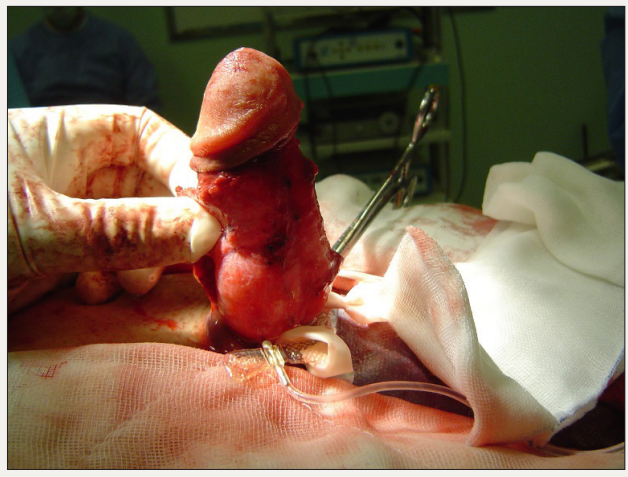

Figure 6: 


\section{Place of the technique in the contemporary treatment}

Penil prosthesis implantation is the standard surgical treatment for severe PD with concurrent ED [5]. Therefore, combined penil revascularization with penil curvature correction surgery should be regarded as an option, only in case of the prosthesis implantation procedure is not feasible, or when the patient wants to avoid prosthesis. Both the short- and the long-term results of the prosthesis implantation is superior. Also, the most comprehensive case series of the combination surgery comprises a very-selected patient cohort by a single surgeon, which brings an evidence that is inferior to the multi-center results of prosthesis implantation. However, the total success of the procedure is not negligible [1,3].

Comparing the combination surgery vs. phosphodiesterase- 5 (PDE-5) inhibitors, intraurethral or intracavernosal treatments is not possible because the lack of evidence, and the difference of the main indications of the treatment modalities. While PDE-5 inhibitors and topical therapies are used in the primary and the secondary management of ED, respectively, the combination surgery is an alternative of penil prosthesis implantation, which is the third step of the management.

Drawing an exact head-to-head comparative conclusion seems like not possible. We can conclude that the penil prosthesis should be offered to the patients as the standard treatment of severe PD with concurrent ED that is not responding to medical treatments. However, while the patients seek an alternative modality, the combination of penil revascularization and penile straightening procedures can be offered with acceptable outcomes in the specialized centers. A careful patient selection and experience on the vascular micro-anastomosis techniques are the key-points of the good outcomes. Comparing the utility of the epigastric vessels with the other donor sites is not feasible due to non-standard fashion of the technique. However, considering the well-known usage of several veins as donor sites in the patching procedures, we foresee that the epigastric vessels shall provide the anatomical closure of the tunical defect.

\section{Take home messages}

Combined penile revascularization and penile curvature correction surgery is a surgical option for patients with PD and concurrent vasculogenic erectile dysfunction. For small corporal defects, using epigastric vein as a graft is feasible during the surgery. We report two cases and short-term results to show feasibility of epigastric vein utilization in combined penile revascularization and penile curvature correction surgery. Possible advantages of this technique are avoiding lower limb incision and shortening operation time. Further studies comparing this approach with different grafting techniques are needed to decide whether this approach has a significant advantage.

\section{Acknowledgement}

All authors participated in the surgical procedures, and preparation of the published paper

\section{Conflict of Interest}

The authors declare no conflict of interest.

\section{References}

1. Kayigil O, Okulu E (2013) The combination of penile revascularization surgery with penile corrective techniques as an alternative to prosthesis implantation in patients with Peyronie's disease having ED: preliminary results. Int J Impot Res 25(5): 166-171.

2. Meuleman E, Jiang X, Holsheimer J, Wagner G, Knipscheer B, et al. (2007) Corpus cavernosum electromyography with revised methodology: an explorative study in patients with erectile dysfunction and men with reported normal erectile function. J Sex Med 4(1): 191-198.

3. Kayigil O, Okulu E, Akdemir F, Cakici OU (2017) The combination of penile revascularization surgery with penile corrective techniques as an alternative to prosthesis implantation in patients with peyronie's disease accompanied by erectile dysfunction: Long-term results. Int J Impot Res 30(2): 71-78.

4. Egydio PH, Sansalone S (2008) Peyronie's reconstruction for maximum length and girth gain: Geometrical principles. Adv Urol 2008: 205739.

5. Hatzimouratidis K, Giuliano F, Moncada I, Muneer A, Salonia A, et al. (2017) EAU Guidelines on male sexual dysfunction. presented at the EAU Annual Congress London, EAU Guidelines Office, Arnhem, Netherlands.

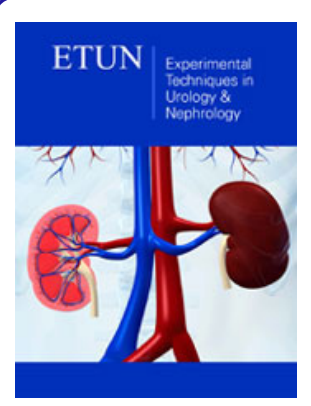

Experimental Techniques in Urology \& Nephrology

\section{Benefits of Publishing with us}

- High-level peer review and editorial services

- Freely accessible online immediately upon publication

- Authors retain the copyright to their work

- Licensing it under a Creative Commons license

- Visibility through different online platforms 